\title{
Wheel-Running Activity Rhythms in Two Inbred Strains of Laboratory Rats Under Different Photoperiods
}

\author{
URSULA SIEBERT ${ }^{1}$ AND FRANZISKA WOLLNIK \\ Department of Biology, University of Konstanz, P.O. Box 5560, 7750 Konstanz, FRG
}

Received 11 April 1991

\begin{abstract}
SIEBERT, U. AND F. WOLLNIK. Wheel-running activity rhythms in two inbred strains of laboratory rats under different photoperiods. PHYSIOL BEHAV 50(6) 1137-1143, 1991. - Wheel-running activity patterns were studied in two inbred rat strains (ACI/Ztm and LEW/Ztm) under 24-h light-dark cycles with various photoperiods. The ACI strain was characterized by a unimodal activity pattern, whereas the LEW strain exhibited a multimodal activity pattern consisting of two activity bouts about 3-5 h apart. Harmonic spectral analyses and chi square periodograms revealed strain-specific differences in the characteristic rhythmic components of the activity pattern. The ACI strain showed only a strong 24-h rhythm, whereas the LEW strain showed additional rhythmic components with periods of $6,4.8$, and $4 \mathrm{~h}$. Except in very young rats, these strain specific patterns were not affected by an experimental lengthening of the dark period. However, differences between the two strains were found in the free-running period and in the mode of entrainment. Our results indicate that the multimodal activity pattern of the LEW strain is not due to an ultradian bout oscillator, instead it may be the result of a unique coupling of multiple circadian oscillators.
\end{abstract}

Circadian and ultradian rhythms Strain differences Entrainment Multioscillatory system

CIRCADIAN rhythms of mammals are assumed to be generated by a self-sustained multioscillatory system in or close to the suprachiasmatic nucleus in the hypothalamus $(16,19)$. Under natural as well as laboratory conditions, these rhythms are entrained by the environmental LD cycle and assume a steady-state phase relationship to this external time cue (14). Characteristic features of circadian rhythms, such as the free-running period $(\tau)$, are not acquired by an organism during ontogeny, but are inherited (11).

Laboratory rats are widely used for genetical studies because there is a great number of inbred strains with differences in the genetic background (8). Therefore, inbred strain comparisons provide a convenient method for investigating the genetic nature of circadian rhythms. Since individuals from the same inbred strain are genetically identical, differences between them must reflect environmental effects or errors of measurement. This means that differences between animals from different strains will exceed the differences between animals from the same strain only if the strains are genetically different.

Using this approach, previous studies have demonstrated genetically based differences in the activity pattern of various commonly available inbred strains of rats $(7,20)$. Based on these results, two of these inbred strains, $\mathrm{ACI}$ and LEW, were selected for the present study. The strain ACI exhibits a strong unimodal activity pattern with a high activity level, clear onsets of activity, and distinct differences between activity and rest time. The strain LEW, on the other hand, shows a multimodal activity pattern characterized by two or sometimes even three separate activity bouts about $3-5 \mathrm{~h}$ apart. The same strain specific patterns have also been observed in the daily rhythms of body temperature, heart rate (6), food intake, and oxygen consumption (5). Period analysis of the activity pattern of the LEW strain revealed ultradian components with periods of 4 and $4.8 \mathrm{~h}$ in addition to the 24-h rhythm. The fact that these additional periodic components are subharmonics of the 24-h rhythm can be explained either with an ultradian modulation of the activity pattern or with a unique phase coupling of multiple circadian oscillators.

Preliminary evidence of the multioscillatory nature of the activity pattern of the LEW strain is given by a strong correlation between onset and offset of each of the two activity components, but no significant correlation between the two bouts (20). For a true ultradian modulation, however, the correlation between the two bouts should have been of the same magnitude as the correlation between onset and offset within each bout.

To further evaluate the mechanisms underlying the multimodal activity pattern of the LEW strain, the present study examined the effect of 24-h LD cycles of different photoperiods on the phase relationship between activity bouts. The strain $\mathrm{ACI}$ was chosen as a representative for the typical unimodal activity pattern of laboratory rats.

\section{Animals and Housing}

METHOD

Male rats of the inbred strains $A C L / \operatorname{tm}(N=5)$ and LEW $(\mathrm{N}=5)$, originally obtained from the Central Animal Laboratory

\footnotetext{
${ }^{1}$ Requests for reprints should be addressed to Ursula Siebert, Fakultät für Biologie, Universität Konstanz, Postfach 5560, W-7750 Konstanz 1, Fed. Rep. Germany.
} 
at the Hanover Medical School (FRG), were bred and raised in our laboratory under controlled environmental conditions (12:12 h light:dark cycle, lights on at 0700 , room temperature $22 \pm 1^{\circ} \mathrm{C}$ ). At the beginning of the experiment, the animals were $38(\mathrm{ACl}$ $\mathrm{N}=5), 28(\mathrm{LEW} \mathrm{N}=2$ ) and $36(\mathrm{LEW} \mathrm{N}=3)$ days of age. The animals were placed in individual cages (Macrolon Type IV. $35 \times 55 \times 20 \mathrm{~cm}$ ) with constant free access to food, water, and a running wheel (diameter $35 \mathrm{~cm}$. width $10 \mathrm{~cm}$ ). The cages were placed randomly in a cage rack. Light intensity at the level of the cage top was 100-200 lux. Wheel-running activity was monitored under entrainment to different photoperiods for a total of 24 weeks. The initial lighting regime consisted of $18 \mathrm{~h}$ light and $6 \mathrm{~h}$ dark (LD 18:6). The dark period was symmetrically increased by two hours every 3 weeks until a maximal dark period of $18 \mathrm{~h}$ was reached (LD 6:18). The experiment concluded with 3 weeks continuous darkness (DD) and 3 weeks LD 12:12. Prior to the experiment, wheel-running activity of the 3 older LEW rats had been monitored for 3 weeks under LD 14:10 and for another 3 weeks under LD 16:8. The animal room was entered 2-3 times a week at random times during the day to check the water bottles and food supply and to clean the cages.

\section{Data Collection and Analysis}

Three magnetic reed switches were attached to the axle of each running wheel so that one complete wheel revolution resulted in three electrical impulses. These impulses were read online by a microcomputer and stored on disk every $5 \mathrm{~min}$. All subsequent calculations were based on these 5-min counts. Event records were visualized as double plots with a resolution of 20 min. For an example see Fig. 1. Maximum height of a 20-min bin indicates more than 200 impulses (i.e., an average of $10 \mathrm{im}$ pulses/min). while a blank space indicates fewer than $20 \mathrm{im}$ pulses (i.e.. an average of 1 impulse/min).

The presence of periodic components was tested using the "chi square periodogram" (18) and the "harmonic spectral analysis" $(10.13)$ calculated for the whole 21 day long period as well as for the last 7 days of each lighting regime. These two approaches of period analysis are based on different statistical models, and results of both methods were always compared to verify the reliability of the analyses. Both techniques and their application to circadian and ultradian rhythms have been described in greater detail elsewhere (21), and have been tested with artificial data of different periods, amplitude, and waveforms in the presence of different levels of noise (17).

Additional parameters calculated from the activity recordings include: 1) amount of activity: the total number of impulses within a 24-h period, given as impulses/day; 2) duration: total length of all 5-min intervals within $24 \mathrm{~h}$ containing more than 5 impulses, given in min/day; 3 ) maximal activity: largest number of counts within a single 5-min interval. Since it was difficult to visually determine the onsets and offsets of activity, and the peak of each individual activity bout from the activity plots, data of the last 7 days of each lighting regime were averaged and further smoothened using a moving average. Onset of activity was then defined as the start time of the first block of at least 6 consecutive 5 -min intervals with at least 5 impulses that was not separated from the the next block of activity by more than 10 minutes. Offset of activity was defined as the end time of the last in a sequence of such blocks. The following phase differences were calculated: $\psi_{1}=$ time of lights off to time of the first activity peak: $\psi_{2}=$ time of the second activity peak to time of lights on; $\psi_{1-2}=$ time of the first activity peak to time of the second activity peak; $\psi$ lights off to activity onset; $\psi$ lights on to activity onset; wlights off to activity offset: wlights on to activity offset.

Differences between strains and lighting regimes were as sessed using standard procedures of variance analysis IANOVA. SAS). Post hoc comparisons were made using multiple $t$-tests.

RESULTS

\section{Wheel-Running Activity Pattern}

Activity records of two typical animals of each strain are shown in Fig.1. The wheel-running activity of ACI rats showed a generally unimodal pattern, whereas LEW rats exhibited a more bimodal activity pattern with two short activity bouts about 3-5 h apart from each other. Despite the stepwise decrease of the photoperiod, these strain-specific activity patterns remained stable throughout the experiment, and the temporal spacing between the two activity bouts of the LEW rats was not affected by the different lighting regimes. Only 2 LEW rats (e.g., LEW \#3. Fig.1) showed an additional bout of activity that appeared to emerge from the second activity bout during LD 14:10 (week 6-9) and LD 12:12 (week 10-12). However, this third activity bout was never as prominent as the two other activity bouts and was never established under constant dark conditions. In one animal (LEW No. 10, Fig.1), the second peak disappeared twice for several days during LD 16:8 and DD. More dramatic changes of the activity pattern were observed in young LEW rats kept under initial lighting schedules of LD 14:10 and LD 16:8 (Fig. 2). These animals also established a bimodal activity pattern, but the onsets of the two activity peaks were up to $8 \mathrm{~h}$ apart from each other (Fig. 2). The characteristic spacing of 3-5 h between the two peaks was established during LD 16:8 and subsequently did not change under any lighting condition. The activity pattern of the ACI rats was also divided into several activity bouts. However, these bouts were generally shorter, more variable, and not separated by long time spans without any wheel-running activity.

\section{Period Analysis}

Harmonic spectral analyses (Fig. 3) and chi square periodograms revealed different characteristic rhythmic components in the activity pattern of the two strains. The activity pattern of $\mathrm{ACI}$ rats showed only one strong component with a period of 24 $\mathrm{h}$ and an additional rather weak component with a period of 12 h. In contrast, the activity pattern of the LEW strain was characterized by additional components with periods of $6,4.8,4$, and $3 \mathrm{~h}$ corresponding to the temporal organization of the two activity bouts. ANOVA of spectral estimates resulting from harmonic spectral analysis revealed minor changes in the amplitude of the 24-h peak for the ACI strain but not for the LEW strain. In the LEW strain, however, the amplitudes of the ultradian components, i.e., $12,4,3$, and $2 \mathrm{~h}$, increased significantly during the experiment. Furthermore, both methods of period analysis demonstrated a systematic shift of the most prominent ultradian components in the activity pattern of the LEW strain. Period analyses of the first two lighting regimes (LD 18:6, LD 16:8) showed the highest amplitudes for periods of 6 and $4.8 \mathrm{~h}$. Towards the end of the experiment, however, periods of 4,3 , and $2 \mathrm{~h}$ were more prominent.

The characteristic rhythmic components of both strains persisted under constant darkness (DD). The chi square periodogram revealed a significant difference $(p \leq 0.01)$ between the free-running period of the $\mathrm{ACI}(\tau=24.27 \pm 0.03 \mathrm{~h} \mathrm{SE})$ and the LEW strain $(\tau=24.1 \pm 0.02 \mathrm{~h} \mathrm{SE})$. 

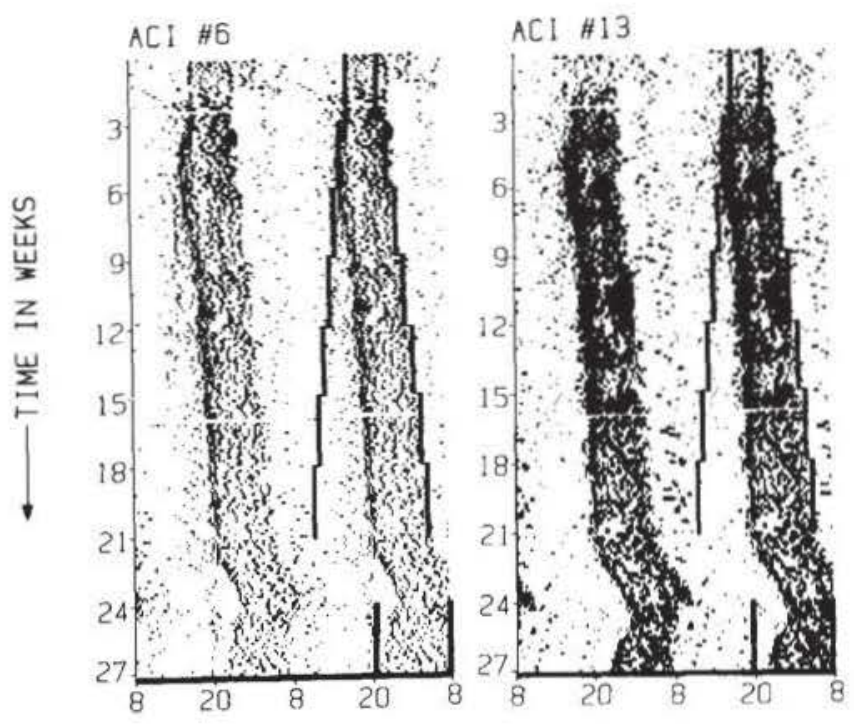

LD 18: 6

LD 16:8

LD 14:10

LD 12: 12

LO 10: 14

LO 8: 16

LD 6: 18

DD

LD 12: 12
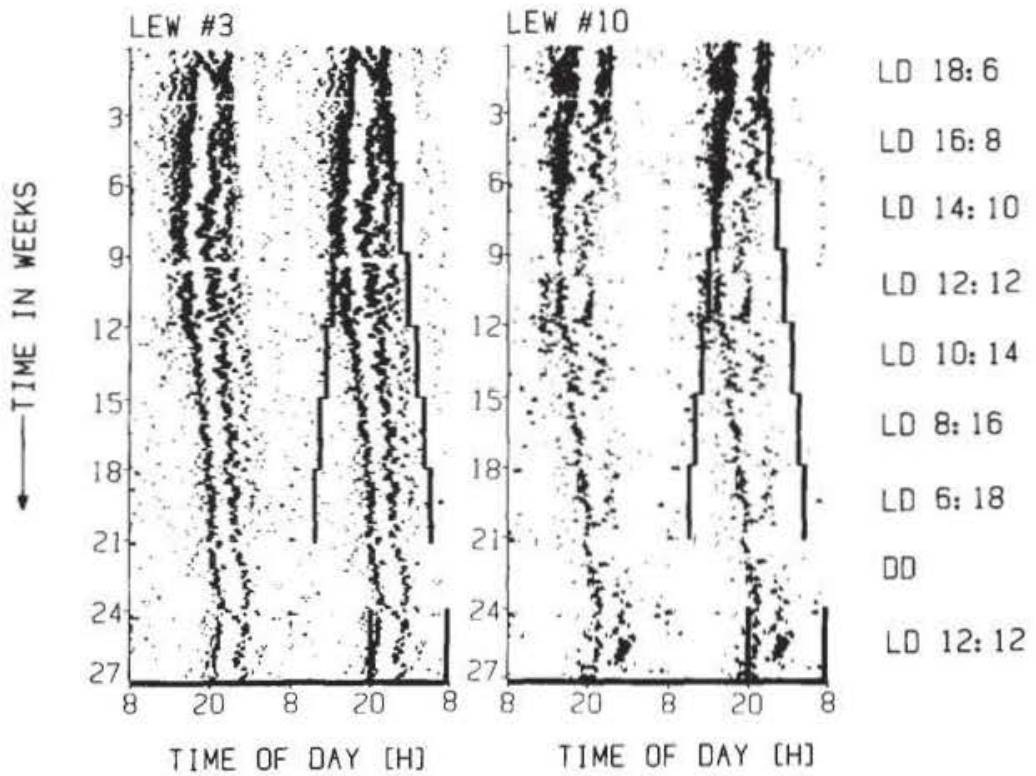

FIG. 1. Double plotted wheel-running activity records of $2 \mathrm{ACI}$ and 2 LEW rats maintained under different photoperiods. Numbers on the vertical axis denote weeks of experiment, numbers on the horizontal axis denote daytime hours. Days with data missing due to power failure or system errors are shown as blank lines. The black bars on the right side of each plot indicate beginning and end of the dark period. From top to bottom, the following lighting regimes were presented for a period of 21 days each: LD 18:6, LD 16:8, LD 14:10, LD 12:12, LD 10:14, LD 8:16, LD 6:18, DD.

\section{Level of Activity}

Figure 4 summarizes changes in the amount of wheel-running activity for each strain. In general, $\mathrm{ACl}$ rats were more active than LEW rats, (ACI: $1219 \pm 166$ impulses/day; LEW: $914 \pm 87$ impulses/day, $p \leq 0.01$ ). At the beginning of the experiment (day 1-21), however, LEW rats were significantly more active than $\mathrm{ACI}$ rats. The amount of activity did not change in the $\mathrm{ACI}$ strain, but decreased continuously in the LEW strain without significant differences between consecutive lighting regimes. From LD 10:14 (week 12) onwards, the amount of wheel-running ac- tivity was significantly higher in ACI rats. The maximal values of activity $\left(A_{\max }\right)$ were slightly smaller for $\mathrm{ACI}$ rats (ACI: $67 \pm 3$ impulses $/ 5 \mathrm{~min}$; LEW: $74 \pm 3$ impulses $/ 5 \mathrm{~min}$ ). $\mathrm{A}_{\max }$ of the LEW strain decreased during the experiment. No significant difference was found in the duration of activity, i.e., ACI and LEW rats spent about the same amount of time in the wheel (ACI: $232 \pm 106$ $\mathrm{min} /$ day; LEW: $230 \pm 61 \mathrm{~min} /$ day).

\section{Activity Onset and Offset}

Figure 5 depicts changes in the time ( $\alpha$ ) between activity on- 

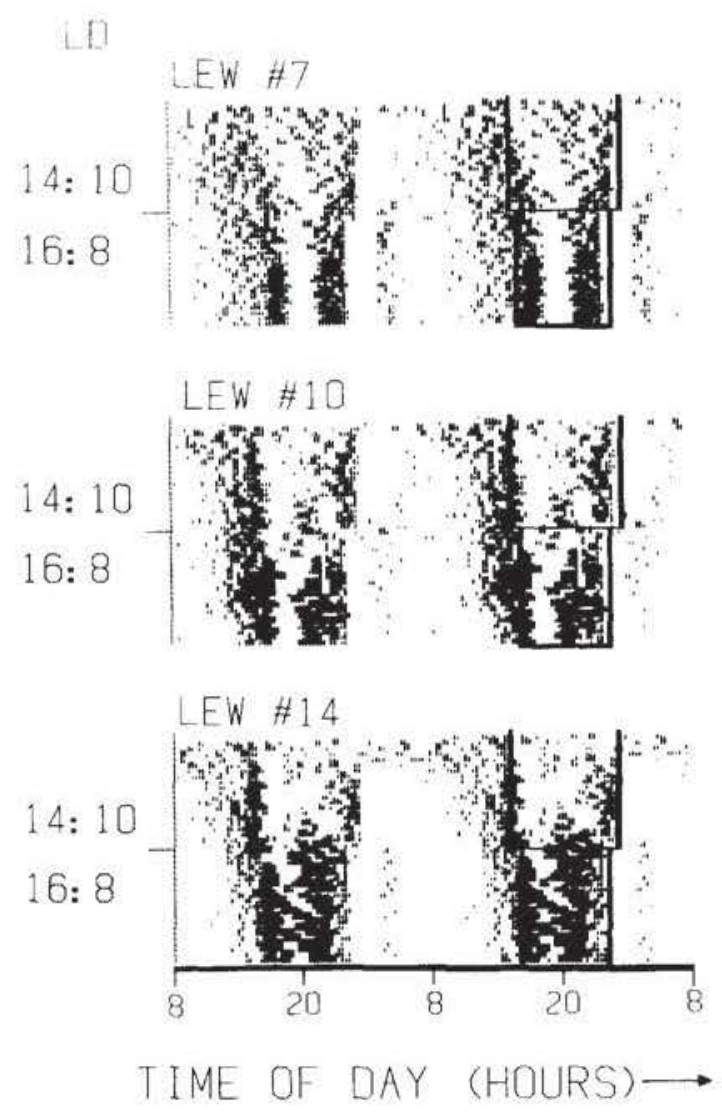

FIG. 2. Activity records of 3 LEW rats maintained for 3 weeks each under LD 14:10 and LD 16:8. For further explanations see Fig. 1.

set and offset and in the phase difference $\left(\psi_{1-2}\right)$ between the two activity peaks of the LEW strain during the course of the experiment. $\alpha$ was significantly $(p \leq 0.01)$ longer in the ACI strain $(9.36 \pm 0.27 \mathrm{~h} \mathrm{SE})$ than in the LEW strain $(8.08 \pm 0.43 \mathrm{~h} \mathrm{SE})$. In the ACI strain, $\alpha$ increased only during DD (Fig. 5, upper panel), while in the LEW strain $\alpha$ decreased continuously (Fig. 5 , lower panel).

Visual inspection of the activity records (Fig. 1) revealed obvious differences between the ACI and the LEW strain with respect to their entrainment under different photoperiods. In the $\mathrm{ACI}$ strain, activity onset was tightly locked to the end of the dark period in one rat from the beginning of the experiment and in four other rats from LD 14:10 onwards. In contrast, the activity onset of all LEW rats was locked to the beginning of the dark period until LD 12:12 and in two rats even until LD 10:14. These different modes of entrainment are also apparent in the different $\psi$ values shown in Fig. 6: activity onset of the ACI strain displayed a stable phase relationship with the end of the dark period throughout the whole experiment, whereas the activity onset of the LEW strain was locked to the beginning of the dark period in long photoperiods and to the end of the dark period in short photoperiods. During very short nights (LD 18:6), the activity onset of the LEW rats occurred several hours before lights off. However, the total amount $(70 \%)$ of activity during the dark phase was still larger than during the light phase $(30 \%)$. $\mathrm{We}$, therefore, considered these animals to be nocturnal under LD 18:6. The phase difference $\left(\psi_{1-2}\right)$ between the two activity bouts of the LEW strain did not increase under longer dark peri- ods. The highest $\dot{\psi}_{1-2}$ was found during LD 10:14 in young LEW rats $(6.03 \pm 0.97 \mathrm{~h} \mathrm{SE})$.

\section{DISCUSSION}

The present study demonstrated strain-specific activity patterns for $\mathrm{ACl}$ and LEW rats that were surprisingly consistent under different photoperiods. In both strains, the general pattern of wheel-running activity was almost identical with the one previously observed under LD 12:12 entrainment (20). ACI rats had a unimodal activity pattern characterized by a constant high level of activity with clear onsets and offsets. In contrast, LEW rats showed a multimodal activity pattern with two rather short activity bouts about 3-5 hours apart from each other. Previous studies have shown a similar pattern in the daily rhythms of overall activity as measured with an Animex-like system (7), body temperature and heart rate (6), and food intake and oxygen consumption (5).

Experimental lengthening of the dark period from LD 18:6 to LD 6:18 did not cause dramatic changes in the activity patterns of the two strains. Period analyses of the activity patterns revealed that lighting regimes had only minor effects on the characteristic frequencies. For the LEW strain, in particular, a change in the phase relationship between the two activity bouts could be observed only in very young rats. Ultradian components of 6 and $4 \mathrm{~h}$ were more prominent during the first weeks of the experiment (LD 18:6, LD 16:8), whereas shorter periods were more prominent towards the end of the experiment. We believe that this effect was due to the age of the animals rather than to photoperiod, because a similar shifting of the major rhythmic components has also been demonstrated under long-term LD 12:12 entrainment. Ultradian periods of $6,4.8,4$ and $3 \mathrm{~h}$ persisted under DD demonstrating the endogenous nature of these rhythmic components.

In their studies on hamsters and mice, Aschoff and co-workers (1-4) showed that lengthening of the dark period causes an increase in activity time $(\alpha)$ as well as in the phase relationship between the two major activity bouts of bimodal activity patterns. These observations led to the hypothesis of two separate circadian oscillators controlling the rhythms of different activity components (15). The two oscillators are presumed to be differentially coupled to lights-off (i.e., evening) and lights-on (i.e.. morning) as well as to each other. In the present study, lengthening of the photoperiod neither resulted in an increase of activity time in the LEW strain nor in any systematic change of the temporal spacing of the activity bouts. A longer interval between the two activity bouts could only be observed in very young LEW rats under LD 14:10 and LD 16:8. Once the optimal phase relationship of 3-5 h had been established, a strong coupling between the two activity components seemed to prevent any further modulation of the activity pattern by the photoperiod. Therefore, the present results do not exclude the hypothesis of a "morning" and "evening" oscillator generating the activity pattern of LEW rats. Further studies are necessary to verify whether the upbringing of LEW rats under different photoperiods will affect the temporal organization of the activity pattern during adulthood.

The observed stability of the bimodal activity pattern of the LEW strain in prolonged nights and the extended phase relationship between the two activity peaks in young LEW rats contradict the hypothesis of an ultradian bout oscillator. Further evidence against a true ultradian pacemaker has been presented in a previous study (22) demonstrating that, unlike the ultradian rhythms of feeding and wheel-running in voles (9), the activity pattern of LEW rats does not persist after complete SCN lesions. Rather, 


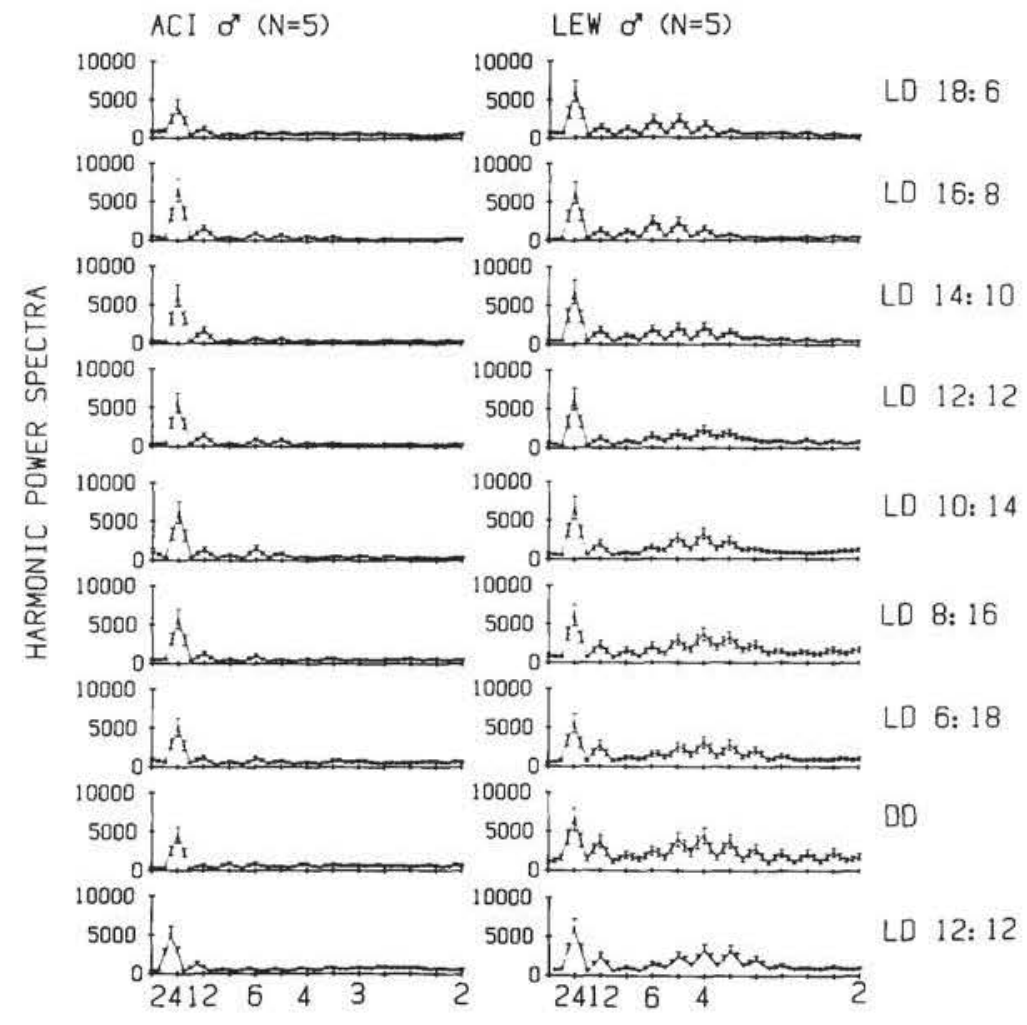

FIG. 3. Harmonic spectral analysis of wheel-running activity under different photoperiods for strains ACI (left panel, $N=5$ ), and LEW (right panel, $N=5$ ). Power spectra of individual animals were pooled for each strain. The $95 \%$ confidence limits of spectral estimates are plotted as vertical lines.

the present results support the altemative hypothesis of a multioscillatory circadian system. The occurrence of a third activity peak in some cases indicates that more than two oscillators may be involved in the organization of the activity pattern.

So far, the neuronal basis for the observed strain differences is unknown. A previous study (20) demonstrated a strong correlation between the intensity of wheel-running activity and the coherence of the 24-h rhythm, suggesting that the multimodal activity pattern of the LEW strain is a direct result of the reduced activity level. In the present study, however, young LEW rats ran significantly more than ACI rats of about the same age and still displayed a multimodal activity pattern. These results indicate that the unimodal pattern of the ACI strain is not simply caused by a "filling of the trough," and that the multimodal activity pattern of the LEW strain is not attributable to lack of activity feedback to the circadian system. Furthermore, it seems unlikely that the observed strain differences are simply due to the different pigmentation, because previous investigations of other inbred rats had found a typical unimodal activity pattern in both pigmented and albino rats (7).

An interesting finding of the present study is the different mode of entrainment observed in ACI and LEW rats. As demonstrated by periodogram analyses, wheel-running activity of both inbred strains, ACI and LEW, was clearly entrained during the third week of each photoperiod. There were obvious differences between the two strains with respect to the interindividual variability of activity onset and the phase angle difference between activity and entraining LD cycle. The activity rhythm of $\mathrm{ACI}$ rats was tightly locked to the end of the dark period (lights-on) during all photoperiods, whereas in the LEW strain, activity onset was more variable and seemed to be coupled to the beginning of the dark period in long photoperiods (LD 18:6, LD 16:8, LD 14:10, LD 12:12). The early onset of activity under LD 18:6 can be explained by a preference for the slightly

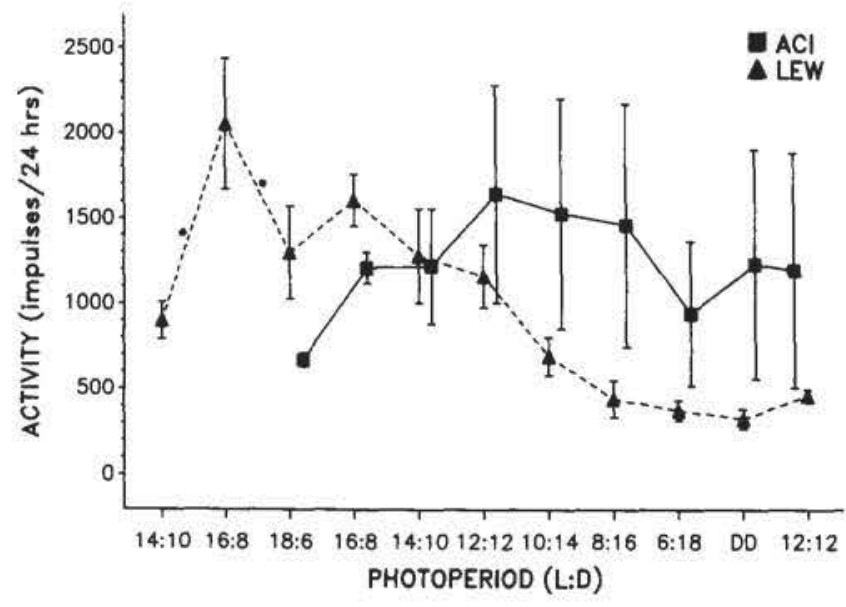

FIG. 4. Amount of wheel-running activity in ACI $(\mathrm{N}=5)$ and LEW $(\mathrm{N}=5)$ rats under different LD regimes. Amount of activity was defined as the total number of impulses per day. Graphs show means $\pm S E$ for $\mathrm{N}=5$ animals of each strain. 

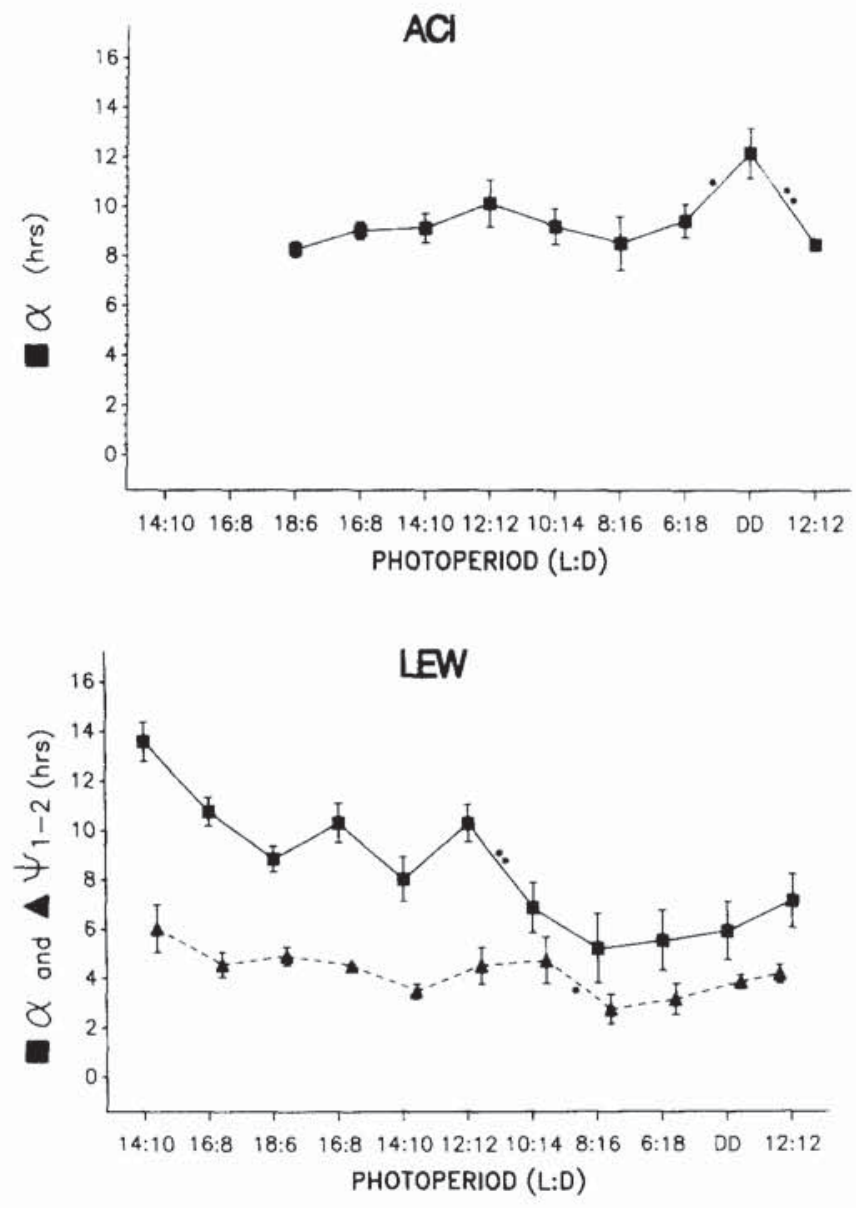

FIG. 5. Time $(\alpha)$ between activity onset and offset for $5 \mathrm{ACI}$ rats (upper panel) and for 5 LEW rats (lower panel) under different photoperiods. The lower panel also shows the phase difference between the two activity peaks in LEW rats. Vertical bars indicate means \pm SE. Significant changes within strains between two consecutive photoperiods are indicated by asterisks $\left({ }^{*} p<0.05,{ }^{* *} p<0.01\right)$.

darker interior of the wheel. Alternatively, the compression of the phase relationship between the two activity bouts might reach its limit in very short nights and the animals might be forced to run during the last hours of the light phase. Assuming a freerunning period shorter than or close to $24 \mathrm{~h}$, a greater variability in the phase angle difference between activity and the entraining LD cycle is predicted for the LEW strain by the nonparametric model of entrainment proposed by Pittendrigh and Daan (14). Rats normally have a mean $\tau$ longer than $24 \mathrm{~h}$, and their PRC is characterized by a larger delay region (12). Additional studies will be necessary to clarify whether the observed differences in the mode of entrainment in the ACI and LEW strains are due to differences in their PRCs or to differences in the sensitivity of their visual systems. Establishing the PRC of the LEW strain could furthermore help to understand its multimodal activity pattern. Specifically, it would be interesting to see whether light
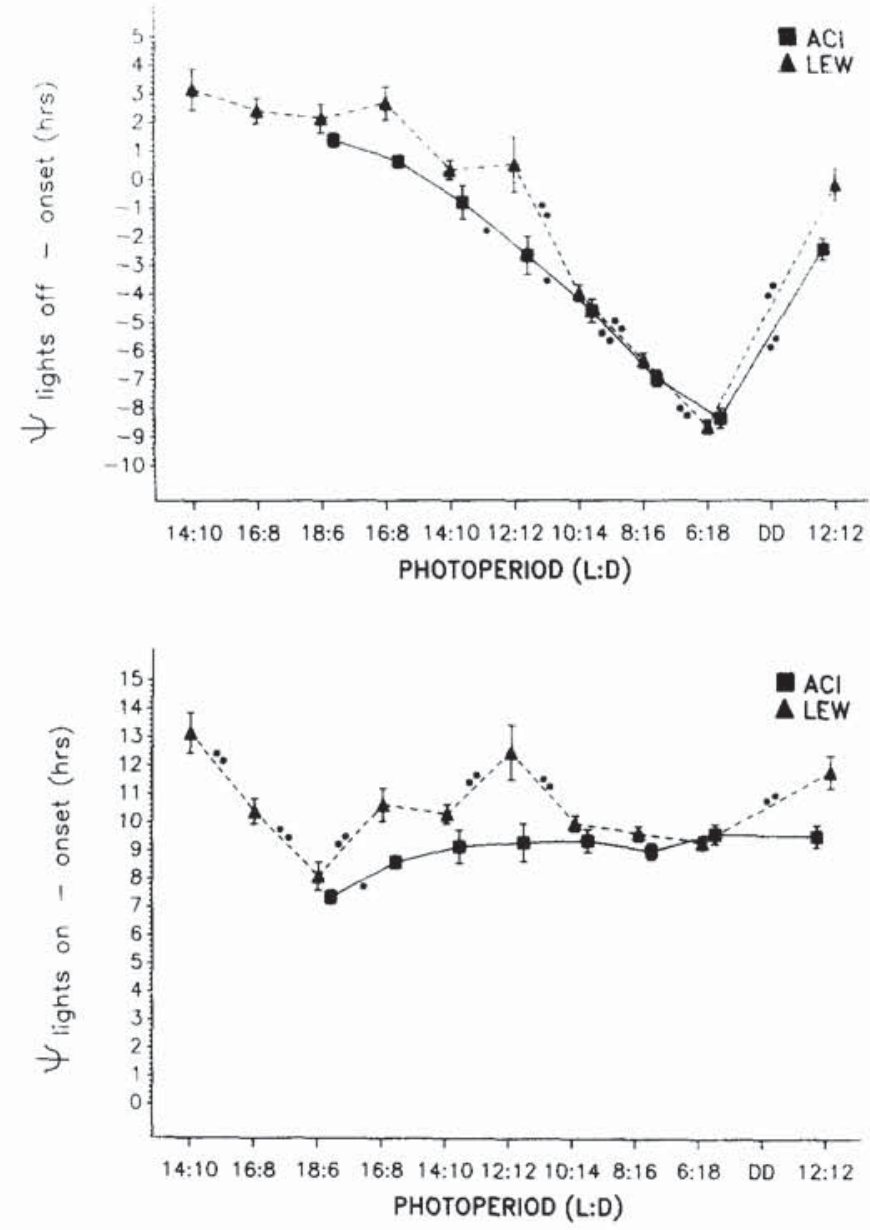

FIG. 6. Temporal relationships between activity onset and lights-off (upper panel) and between activity onset and lights-on (lower panel) under different photoperiods for $5 \mathrm{ACI}$ rats and for 5 LEW rats. Vertical bars indicate means \pm SE. Significant changes within strains between two consecutive photoperiods are indicated by asterisks. $\left({ }^{*} p<0.05,{ }^{* *} p<0.01\right)$.

pulses set in between the two activity bouts would shift them in different directions.

In summary, the present study suggests that the wheel-running activity pattern of LEW rats is generated by a multioscillatory system rather than an ultradian oscillator. The true mechanism underlying the functional complexity of the circadian system of the LEW rats remains to be determined. Further experiments, such as the PRC or different T-cycles, may help to elucidate the underlying mechanism of the complex activity pattern of this inbred strain.

\section{ACKNOWLEDGEMENTS}

The authors wish to thank S. Daan for his valuable comments on an earlier version of the manuscript. This research was supported by a grant from Deutsche Forschungsgemeinschaft (Wo-354/3-1).

\section{REFERENCES}

1. Aschoff, J. Spontane lokomotorische Aktivität. In: Helmcke, Y. G.; von Lengeken, H.; Starck, D., eds. Handbuch der Zoologie 8. Berlin: Walter de Gruyter; 1962:1-76.

2. Aschoff, J. Circadian activity pattern with two peaks. Ecology 47(4):657-662; 1966.
3. Aschoff, J.; Meyer-Lohmann, J. Die 24-Stunden-Periodik von Nagern im natürlichen und künstlichen Belichtungswechsel. Z. Tierphysiol. 11(3):476-484; 1954.

4. Aschoff, J.; Meyer-Lohmann, J. Die Aktivitätsperiodik von Nagern im künstlichen 24-Stunden-Tag mit 6-20 Stunden Lichtzeit. Z. 
vergl. Physiol. 37:107-117; 1955.

5. Büttner, D. Influence of strain specific patterns of locomotor activity on the daily pattern, minimal, mean and maximal oxygen consumption in the rat. Z. Versuchstierkd. 29:121-128; 1987.

6. Büttner, D.; Wollnik, F. Spontaneous short-term fluctuations in the daily pattern of heart rate, body temperature, and locomotor activity in the laboratory rat. Lab. Anim. 16:319-326; 1982.

7. Büttner, D.; Wollnik, F. Strain-differentiated circadian and ultradian rhythms in locomotor activity of the laboratory rat. Behav. Genet. 14(2):137-152; 1984.

8. Festing, M. F. W. Inbred strains in biomedical research. London: Macmillan; 1979

9. Gerkema, M. P.; Groos, G. A.; Daan, S. Differential elimination of circadian and ultradian rhythmicity by hypothalamic lesions in the common vole, Microtus arvalis. J. Biol. Rhythms 5(2):81-95; 1990.

10. Halberg, F.; Panofsky, H. Thermo-variance spectra; Method and clinical illustrations. Exp. Med. Surg. 19:285-309; 1961.

11. Hall, J. C. Genetics of circadian rhythms. Annu. Rev. Genet. 24: 659-697; 1990.

12. Johnson, C. H. An atlas of phase response curves for circadian and circatidal rhythms.

13. Panofsky, H.; Halberg, F. Thermo-variance spectra; Simplified computational example and other methodology. Exp. Med. Surg. 19: 322-338; 1961

14. Pittendrigh, C. S.; Daan, S. A functional analysis of circadian pacemakers in nocturnal rodents. IV. Entrainment: Pacemaker as clock. J. Comp. Physiol. 106:291-331; 1976.

15. Pittendrigh, C. S.; Daan, S. A functional analysis of circadian pacemakers in nocturnal rodents V. Pacemaker structure: A clock for all seasons. J. Comp. Physiol. 106:333-355; 1976.

16. Rosenwasser, A. M.; Adler, N. T. Structure and function in circadian timing systems: Evidence for multiple coupled circadian oscillators. Neurosci. Biobehav. Rev. 10:431-448; 1986.

17. Siebert, U.; Wollnik, F. Statistical analysis of ultradian rhythms: A comparison of different methods of time series analysis. In: Second meeting society for research on biological rhythms. abstract:62; 1990.

18. Sokolove, P. G.; Bushell, W. N. The chi-square periodogram: Its utility for analysis of circadian rhythms. J. Theor. Biol. 72:131-160; 1978.

19. Turek, F. W. Circadian neural rhythms in mammals. Annu. Rev. Physiol. 47:49-64; 1985.

20. Wollnik, F. Strain differences in the pattern and intensity of wheel running activity in laboratory rats. Experientia 47:593-598; 1991.

21. Wollnik, F.: Döhler, K. D. Effects of adult or perinatal hormonal environment on ultradian rhythms in locomotor activity of laboratory LEW/Ztm rats. Physiol. Behav. 38(2):229-240; 1986.

22. Wollnik, F.; Turek, F. W. SCN lesions abolish ultradian and circadian components of activity rhythms in LEW/Ztm rats. Am. J. Physiol. 256:R1027-R1039; 1989. 